MS36-P9 Hydrogen bonding in a

benzimidazole derivate - what is in a name

? A combined use of powder diffraction, solid state and molecular DFT study.

Lubo Smrcok ${ }^{1}$, Pavel Mach ${ }^{1}$

1. Institute of Inorganic Chemistry, Slovak Academy of Sciences, Dubravska cesta 9, SK-845 36 Bratislava, Slovak Republic

email: uachsmrk@savba.sk

Apart for obvious electrostatic interactions the molecules of methyl 3-((benzimidazol-4(7)-yl) amino)-2-cyano-prop-2-enoate are in the structure (solved from powder diffraction data and refined by high quality energy minimization in the solid state) also held by $\mathrm{N}-\mathrm{H}$...N and $\mathrm{C}-\mathrm{H}$...N hydrogen bonds, remarkably differing in their strengths and roles. To evaluate the strength of these hydrogen bonds in the structure molecular DFT calculations applying dispersion corrected functional B97-D3 (environment-dependent D3 scheme with haVDZ basis set) as well as wave-function based SCS-MP2 method with def2-TZVPP basis set were done. Because wave-function based methods treat intermolecular electron correlation (a quantum-mechanical origin of dispersion interaction) in a different way than DFT does, application of the methods differing in their nature provides a stringent test of consistency of the resulting values. To estimate the interaction energy of infinite chain of N-H..N hydrogen bonded molecules a standard procedure based on extrapolation to infinity was applied. First, the interaction energies in a dimer, a trimer and in a tetramer of molecules of linked by one, two and three N-H...N bonds, were calculated. However, rather than the absolute value of the interaction energy it is the size of one its ingredients, of a dispersion interaction, which is of main interest here. For a dimer this contribution represents $78 \%$ of the interaction energy and this share slightly increases for the trimer $(83 \%)$ and than just marginally for the tetramer $(86 \%)$. Dispersion interaction calculated for pairs of molecules bound by the weaker C-H..N hydrogen bond is at DFT level of theory also relatively large, $\sim 55 \%$. It can be thus concluded that although from pure formal geometrical point of view the ribbons of the molecules in the structure are connected by hydrogen bonds, it should be clearly understood that in addition to the "pure" H-bond mechanism of interaction, it is dispersion interaction, which plays important role.

This work was supported by the Slovak Research and Development Agency under the contract No. APVV-0038-11.

Keywords: Weak interactions, hydrogen bonds, DFT, SCS-MP2
MS36-P10 Structural diversity in supramolecular compounds of para-sulfonatocalix[8]arene with phenanthroline

Barbara Lesniewska $^{1}$, Kinga Suwinska ${ }^{2}$, Anthony W. Coleman ${ }^{3}$

1. Institute of Physical Chemistry PAS, ul. Kasprzaka 44/52, PL-01-224 Warszawa, Poland

2. Faculty of Mathematics and Natural Sciences, Cardinal Stefan Wyszynski University, Wóycickiego 1/3,PL-01 938 Warszawa, Poland

3. LMI, Universit_e Lyon 1 CNRS UMR 5615, 43 bvd 11 novembre, 69622 Villeurbanne, France

email: blesniewska@ichf.edu.pl

para-Sulfonatocalix $[n]$ arenes are anionic, water soluble derivatives of calix $[n]$ arenes, which are supramolecular hosts and 'building blocks' in crystal engineering. An important feature of these compounds is their ability to co-crystallize with variety of molecular species. They form a series of complexes with polar and non-polar molecules and/or metal cations both in solution and solid-state. They create a variety of supramolecular architectures such as bilayers, molecular capsules, polymers stabilized by hydrogen bonds, one or two-dimensional coordination polymers, motifs like 'ferris wheel' and 'Russian doll', helical arrays, channels filled with water molecules as well as nanometer-sized spheres or tubes 1 .

Calix[8]arenes themselves may adopt sixteen 'up-down' conformations ${ }^{1}$ and numerous others in which one or more of the aryl rings projects outward from the average plane of the molecule, depending on the functionalization of the macrocycle, the solvent used for the crystallization or the shape and nature of complexed guest molecules. The most common conformation, both in solution and in the solid state, is pleated loop conformation (Fig.1), stabilized by intramolecular hydrogen bonds $\mathrm{O}-\mathrm{H} \cdots \mathrm{O}$.

Calixarenes, due to their diversity and conformational mobility are important receptors in molecular recognition. The goal of this paper is to show structural diversity of para-sulfonatocalix[8]arene in complexes with 1,10-phenanthroline in solid state.

${ }^{1}$ C. D. Gutsche. Calixarenes: An Introduction, 2nd ed., Royal Society of Chemistry, 2008.

${ }^{2}$ F. Perret, V. Bonnard, O. Danylyuk, K. Suwinska, A. W. Coleman. 2006, New J. Chem., 30, 987. 


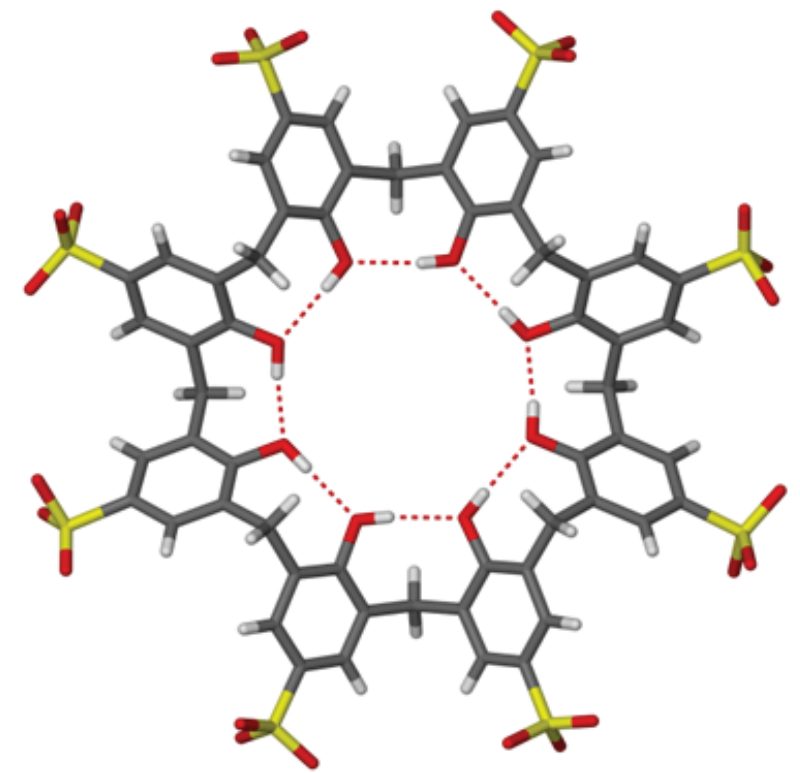

Figure 1. Pleated loop conformation of para-sulfonatocalix[8] arene $^{2}$.

Keywords: calixarenes, host-guest complexes, molecular recognition

\section{MS36-P11 Hydrogen-bonded dimers of resorcinarene-based oxalamido-containing cavitands}

Mario Cetina ${ }^{1,2}$, N. Kodiah Beyeh ${ }^{1}$, Zoran Džolić ${ }^{1}$, Kari Rissanen ${ }^{1}$

1. Department of Chemistry, University of Jyväskylä, P.O. Box 35 , 40014 Jyväskylä, Finland

2. Department of Applied Chemistry, Faculty of Textile

Technology, University of Zagreb, Zagreb, Croatia

email: mcetina@ttf.hr

The rational design of organic compounds and their controlled self-assembly through non-covalent associations to give defined supramolecular nano-structures is a stimulative and innovative approach for the development of smart structures and materials with extensive structural diversity and applications. Recently, much attention has been paid to researches on molecular systems containing self-complementary groups with the potential to construct cages, bowls and capsules $[1,2]$.

Herein, we present the crystal structures of two resorcinarene-based oxalamido-containing cavitands featuring extended sides and self-complementary hydrogen bonding sites that form capsular-like structures. The hydrogen bonding interactions in these structures give rise to the formation of dimers. The self-included dimers are linked by numerous intermolecular interactions so forming firstly one-dimensional polymer structures, which are further linked into three-dimensional networks. In the construction of such supramolecular architectures participates various types of interactions, from strong hydrogen bonds $(\mathrm{N}-\mathrm{H} \cdots \mathrm{O}$, $\mathrm{N}-\mathrm{H} \cdots \mathrm{N})$ to weak $\mathrm{C}-\mathrm{H} \cdots \pi$ interactions which also play important role in their formation.

[1] Beyeh, N.K.; Rissanen, K. (2011) Isr. J. Chem., 51, 769-780.

[2] Jędrzejewska, H.; Wierzbicki, M.; Cmoch, P.; Rissanen, K.; Szumna, A. (2014) Angew. Chem. Int. Ed. 53, 13760-13764.

Keywords: cavitands, resorcinarenes, non-covalent interactions 